\title{
Anti-inflammatory Activity of Extracts and Fractions of Hertia Intermedia
}

\author{
Hakeemullah¹, Muhammad Anwar ${ }^{1}$, Jahangir Khan ${ }^{1}$, Abdul Manan ${ }^{1}$, \\ Naqeebullah Khan ${ }^{2}$, Abdul Hakeem ${ }^{2,}$, Aziz-Ur-Rehman ${ }^{3}$ \\ ${ }^{1}$ Institute of Biochemistry, University of Balochistan, Quetta 87300-Pakistan \\ 2 Department of chemistry, University of Balochistan, Quetta 87300-Pakistan \\ ${ }^{3}$ Bacha Khan University Charsadda, Khyber Pakhtunkhwa 24631-Pakistan
}

\begin{tabular}{ll}
\hline Articles Information & Abstract \\
\hline Received: & This study aims at preparation of extract of medicinal plant Hertia intermedia \\
29.12 .2019 & and determination of their anti-inflammatory activity in it. The plant Hertia \\
Accepted: & intermedia (Root, Stems, leaves and flowers) was collected and was dried under \\
20.04 .2020 & the shade. After drying the plant was subjected to grinding in order to obtain \\
Published: & powdered form. The powder obtained was soaked for the period of $10-15$ days in \\
04.06 .2020 & methanol (CH3OH). The extract obtained was filtered and the remaining solvent \\
& was removed using rotary evaporator. Different fractions of solvent using \\
Keywords: & aqueous dichloromethane, benzene, butanol, chloroform, ethyl acetate and $\mathrm{n}-$ \\
Anti-inflammatory & hexane were prepared using methanol extract of Hertia Intermedia. Methanol \\
Bioactivity & extract, aqueous extract, butanol extract and extract of Hertia intermedia \\
Medicinal plant & showed no bioactivity against inflammatory cell line. Hertia intermedia did not \\
& show any noticeable activity. Hertia intermedia have no bioactivity against \\
\hline
\end{tabular}

DOI: 10.22401/ANJS.23.2.12

* Corresponding author: abduhakeemtareen93@gmail.com

\section{Introduction}

Anti-inflammation is a response of any material, chemical or management against any complication or disease that control, treat manage or act against inflammation or swelling. There are primarily two different types of inflammations, acute and chronic [1]. Robbins acute inflammation could be a fast host response that serves to deliver leukocytes and plasma proteins, like antibodies, to sites of infection or tissue injury e.g. Acute inflammation and Chronic inflammation. Robbins is inflammation of extended period weeks or months in which inflammation, tissue injury and tries at repair co-exist, in varied mixtures [2]. They are used as an analgesic and are referred to as antiinflammatory drugs. Analgesic substances are the drugs or substances that help to alleviate pain and are commonly referred to as pain killers. They act as a remedy against the ache by plummeting inflammation as contrasting to opioids that act on CNS. Opioids are the basic class of analgesics which perform their action by acting on CNS [3]. Inflammation is a defense response of enormous benefit for maintenance of the body homeostasis. The inflammation is a response that may be suitable physiologically and obligatory in the presence of an infection produce by microbes or any other source and damage at cellular as well as at tissues level or stress [4]. Contrary to this, it may be unsuitable due to change or imbalance of homeostasis of body, clinical condition and detrimental when it is released abnormally or without any need. It may result in noxious or damaging effect for the body and in the propagation of ailment that can lead to a serious disease [5] and results in the propagation of ailment that can lead to the serious disease. In actual fact inflammation is the reason of serious concern in diseases like age-related muscular degeneration, Alzheimer's, obstructive pulmonary disease, multiple sclerosis, osteoarthritis, stroke, cardiac disease, and carcinomas [6]. Inflammation is commonly linked with the increase in permeability of the blood vessels and mediator like reactive oxygen species release [7], with the increase in denaturing of amino acids of protein and with modification in cell membrane. Furthermore, the blood cells like leucocyte permeation, granuloma formation or edema also symbolize distinctive indication for presence of inflammation [8]. Moreover, the host retort has been considered to be mainly due to the activation of blood cells like monocytes/macrophages, neutrophils B and T lymphocytes. This type of cells 


\section{Al-Nahrain Journal of Science}

ANJS, Vol.23 (2), June, 2020, pp. 80 - 83

is stimulated and in response they start to produce inflammatory initiators, that are triggered by the release of proteolytic enzymes, arachidonic acid metabolites chemokines, and cytokines, which communally cause the deficit of tissue. This will result in complete degeneration of tissues as well as cells by triggering the diverse degenerative pathways present in the host body [9]. Steroidcontaining drugs and non-steroid containing drugs which are also referred to as the anti-inflammatory drugs are well recognized to manage inflammation as well as pain due to inflammation. Nevertheless, the prolonged and regular use of steroids for a long period of time usually results in small side effects and ailments like hemorrhage, peptic ulceration, gastrointestinal tract dyspepsia and perforation of the vascular system which may lead to the death of the patient in severe cases of perforation, particularly with patients depending with these analgesics [10]. Many medicines derived from plant have been used for a long time without any noticeable side effect or undesirable effects. The development of screening technology has facilitated the discovery of new medicinal plants that are active ingredient and used for the preparation of many analgesic and antiinflammatory drugs [11]. Over $30 \%$ of the w plant species such as leaves, stem and flower, in a steady progression or other, were utilized for remedial decisions.

According to certain concentrates as of not long ago around 120 helpful plants medications of known structure from around 90 types of plans have been researched up until now. Among those restorative specialists the absolute most basic plant drugs incorporate Camptotecin, Digitoxigenin, morphine, atropine, vincristine, vinblastine, Taxol, Podophyllum, lotoxingitxigenin, Digoxigenin, Tubocurarine, codeine, Artemesininaspirin, pilocarpine, Capscicine, Allicin, curcumin, and ephedrine among others. Ethno therapeutic plants are being used for the management of many maladies [12]. Clan Senecioneae, family Compositae involves the significant class Hertia which includes almost 12 species, present in South west Asia and north and south of Africa. Othonnopsis intermedia is the discretionary name of Hertia intermedia which is found in Balochistan in Killa Abdullah, Pishin, Chaman, Quetta, Khanozai. Hertia intermedia is minor bush with blooms of yellow shading. The therapeutic plant, Hertia intermedia is generally used as an analgesic in Pakistan. The phytochemicals present in the plant are known as hertidins. The phytochemicals belong to terpenoids, tannins, flavonoids, coumarins, and steroids [13].

\section{Material and Method}

Identification and Collection of Plant extract:

Hertia intermedia plant was collected from selected areas of province Balochistan such as Killa Abdullah, Pishin and Bostan. It was brought to laboratory of institute of biochemistry university of Balochistan, Quetta for the preparation of samples. The samples were sent to the HEJ laboratory Karachi University for further bioactivity analysis.

\section{Preparation of extract}

The collected plant Hertia intermedia was shade dried for a month, then the dried plant was subjected to grinding to obtained a fine powder. 3 $\mathrm{kg}$ of the powder was weighted and soaked in 10 liters of methanol for 10 - 15 days. The extract obtained after a week was filtered to remove any residual impurities and the liquid was vaporized using rotary evaporator. The extract obtained after vaporization was $104 \mathrm{gm}$ was further fractionated with $\mathrm{n}$-hexane and aqueous medium to obtain $\mathrm{n}^{-}$ hexane fraction $6.5 \mathrm{gm}$ and aqueous fraction $35 \mathrm{gm}$ aqueous fraction was further diluted with butanol to prepare butanol fraction $3 \mathrm{gm}[11,12]$.

\section{Results and Discussion}

To perform the test, four concentrations of Hertia intermedia were used. They are fractions of methanol, aqueous, butanol and n-hexane extracts. All the fractions were screened against inflammatory lines. The concentration used in fraction was $25 \mu \mathrm{mol}$ per assay. The initial phase lasted 90-180 minutes, the point from which the inflammation of the site where the inflammation started and neurotransmitters are released e.g. histamine, serotonin and other neurotransmitters. Following the initial phase, the second phase lasted 270-360 minute. It is correlated with the activation of kinin-like substances such as prostaglandins, proteases and lysosome [10]. Nonesteroidal anti-inflammatory drugs (NSAIDs) are the drug substances which are assumed to be the core drug of choice for the management of ache, discomfort, restlessness and analgesia in people suffering from diseases like gout, osteoarthritis and other inflammatory cases like age-related muscular degeneration in elderly, Alzheimer's diseases, obstructive pulmonary disease, osteoarthritis, gout, cardiac disease and multiple sclerosis etc. One of the studies reveals that estimated $5 \%$ of all doctors' visits in the United 


\section{Al-Nahrain Journal of Science}

ANJS, Vol.23 (2), June, 2020, pp. 80 - 83

States are correlated with prescriptions that advise the usage of non-steroidal antiinflammatory drugs. In the modern era, the NSAIDs are the type of drugs which is noticed to be the mostly and commonly used drugs in normal day life. So, the plant extract and its fractions were screened for anti-inflammatory studies using different cell lines. Natural products are considered to have anti-inflammatory activity with high effectiveness and less toxicity [13].

\section{Hertia intermedia activity against inflammatory cell line}

The extracts are screened against cell lines to check the activity using different concentrations of the extract. The concentration of $25 \mu \mathrm{mol}$ is used for each assay. The prepared dilutions with different solvents (methanol, hexane, butane, aqueous) are coded $\mathrm{MF}, \mathrm{HF}, \mathrm{AF}$, and $\mathrm{BF}$ respectively. These are the fractions which are prepared using different solvent concentrations and are tested against inflammatory cell line. The fractions coded MF and HF exhibited inhibition capacities of $19.7 \%$ and $17.4 \%$ respectively against inflammatory cell line. The fractions coded AF and BF displayed inhibition capacities of $27.3 \%$ and $23.5 \%$ against the same cell line shown in Table 1. The greatest inhibition percentage of aqueous fraction was found among all the samples. The inhibition percentage of butanol fraction was found smaller than $\mathrm{AF}$ but greater than methanol fraction which is in turn greater than that of hexane fraction (Figure 1).

Table 1. Hertia intermedia inhibition rate for inflammatory Cell line.

\begin{tabular}{llll}
\hline $\begin{array}{l}\text { Samples } \\
\text { Coding }\end{array}$ & $\begin{array}{l}\text { Concentr- } \\
\text { ation } \\
\text { (mM) }\end{array}$ & $\begin{array}{l}\text { Inhibi- } \\
\text { tion } \\
\text { age }\end{array}$ & $\begin{array}{l}\text { IC }_{50 \pm} \\
\text { SD }\end{array}$ \\
\hline $\mathrm{MF}$ & 25 & $19.7 \%$ & Inactive \\
$\mathrm{HF}$ & 25 & $17.4 \%$ & Inactive \\
$\mathrm{AF}$ & 25 & $27.3 \%$ & Inactive \\
$\mathrm{BF}$ & 25 & $23.5 \%$ & Inactive \\
\hline
\end{tabular}

MF: methanol fraction

HF: hexane fraction

$\mathrm{AF}$ : aqueous fraction

BF: Butanol fraction

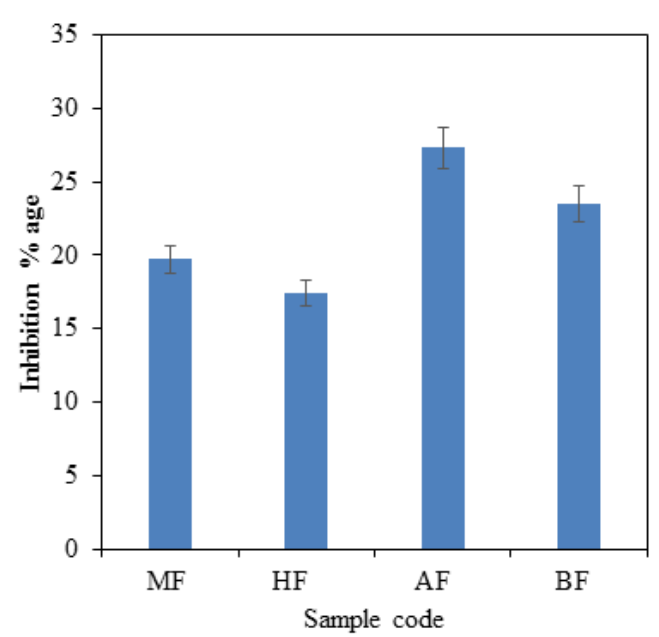

Figure 1. Comparison of inhibition percentage among the samples.

\section{Conclusions}

In this research study, in which the extract of the medicinal plant Hertia intermedia using different dilutions of the solvents were assessed and tested for anti-inflammatory studies against the inflammatory cell lines. After the compilation of the results obtained by our calculations shown that Methanol fraction, aqueous fraction, butanol fraction and $n$-hexane fraction containing Hertia intermedia showed a little bioactivity against inflammatory cell line. As the activity against inflammatory cell line was not noticeable so we concluded that Methanol fraction, aqueous fraction, butanol fraction and $n$-hexane fraction containing Hertia intermedia showed negligible approaching to zero bioactivity against inflammatory cell line.

\section{Acknowledgement}

The authors are very grateful to the institute of Biochemistry, University of Balochistan, Quetta, Pakistan and HEJ laboratory University of Karachi, Pakistan. The authors acknowledged the help taken from others and express their great thankfulness to the University of Balochistan for providing essential laboratory facilities.

\section{References}

[1] Abdelmagid, S.M.; Barbe, M.F.; Safadi, F.F.; "Role of inflammation in the aging bones"; Life Sci. 123, $25-34,2015$.

[2] McCarty, M.F.; "Interleukin-6 as a central mediator of cardiovascular risk associated with chronic inflammation, smoking, diabetes, and visceral obesity: down-regulation with essential fatty acids, ethanol and pentoxifylline"; Med Hypotheses, 52, 465-477, 1999. 


\section{Al-Nahrain Journal of Science \\ ANJS, Vol.23 (2), June, 2020, pp. 80 - 83}

[3] Vane, J. R.; "Botting R.M. Anti-inflammatory drugs and their mechanism of action"; Inflammatory Response, 47, 78-87, 1998.

[4] Gorzalczany, S.; Lopez, P.; Acevedo, C.; Ferraro, G.; "Anti-inflammatory effect of Lithrea molleoides extracts and isolated active compounds"; J. Ethnopharmacol. 133, 994-998, 2011.

[5] Ghudhaib, K. K.; Bakir, S. R.; Hanna, E. R.; "Antimicrobial Activity of Some 1, 3, 4- Thiadiazole Metal Complexes"; Al-Nahrain J. Sci. 17, 1-8, 2014.

[6] Birkedal-Hansen, H.; "Role of cytokines and inflammatory mediators in tissue destruction"; J. Periodont Res. 28, 500-510, 1993.

[7] Ahmed, S. A.; Naji, T. S.; Mohammad, F. I.; "Synthesis, Characterization and Cytotoxic Activity of Some Pyrimidine Derivatives"; Al-Nahrain J. Sci. 16, 84-92, 2013.

[8] Ahmad, M.; Khan, M. P. Z.; Mukhtar, A.; Zafar, M.; Sultana, S.; Jahan, S.; "Ethnopharmacological survey on medicinal plants used in herbal drinks among the traditional communities of Pakistan"; J. Ethnopharmacol. 184, 154-186, 2016.
[9] Jahangir, k. A.; Muhammad, A.; "GC-MS analysis and antileishmanial activity of dichloromethane fraction of Allium cepa (DFAC) in Vitro"; IJPBS. 7, $40-5,2016$

[10] Sultana, N.; Saify, Z.S.; "Naturally occurring and synthetic agents as potential anti-inflammatory and immunomodulants. Anti-Inflammatory AntiAllergy Agents"; Med. Chem.11, 3-19, 2012.

[11] Ramadhan, R. S.; Hussein, Z. F.; Dulaimi, R. K.; "Antibacterial and Anti-inflammatory activities of calendula officinalis growing in Iraq"; World J. Pharm. Res. 4(4), 25-33, 2015.

[12] Maione, F.; Russo, R.; Khan, H.; Mascolo, N.; "Medicinal plants with anti-inflammatory activities"; NPR. 30, 1343-1352, 2016.

[13] Alam, F.; Suleman, S.; "Study of Phytochemistry and Antioxidant Activity of Hertia intermedia (Boiss.) Flowers of Balochistan"; Labela, U. J. Sci. Techl. 92-100, 2015. 